The Astrophysical Journal, 476:L39-L42, 1997 February 10

(C) 1997. The American Astronomical Society. All rights reserved. Printed in U.S.A.

\title{
ON THE BIMODAL MAGNETIC FIELD DISTRIBUTION OF BINARY PULSARS
}

\author{
K. S. CHenG ${ }^{1}$ AND Z. G. DAI ${ }^{2}$ \\ Received 1996 August 19; accepted 1996 November 21
}

\begin{abstract}
We combine the idea of movement of magnetic flux tubes in the stellar interiors with the analysis of crustal physics of accreting neutron stars in low- and high-mass X-ray binaries to explain the bimodal magnetic field distribution of binary pulsars. We propose that this distribution may result from different crustal properties of neutron stars with different accretion rates and amounts of accreted matter. In addition, we may provide an explanation for why the magnetic field strengths of millisecond pulsars with low-mass companions saturate at $\sim 3 \times 10^{8} \mathrm{G}$ when accreted mass exceeds $\sim 0.5 \mathrm{M}_{\odot}$.

Subject headings: dense matter — magnetic fields — pulsars: general — stars: neutron
\end{abstract}

\section{INTRODUCTION}

The magnetic fields of neutron stars play a vital role in radiation mechanisms associated with both rotation and accretion-powered pulsars and with formation and evolution of low-mass X-ray binaries (LMXBs) and millisecond pulsars (Bhattacharya \& van den Heuvel 1991; Phinney \& Kulkarni 1994). As usual, for a pulsar spinning down because of magnetic dipole radiation, the surface magnetic field strength is given by $B_{s}=\left(3 c^{3} I P \dot{P} / 8 \pi R^{6}\right)^{1 / 2}$, where $P$ is the rotation period, $R$ is the radius, $I$ is the moment of inertia, and the angle between the rotation and dipole axes is $\pi / 2$. Thus, the observed pulsars possess surface magnetic fields of strength $\sim 10^{8}-10^{13}$ G. Furthermore, Kulkarni (1992) conjectured that there is a "gap" in the distribution of pulsar magnetic field strengths. This field gap appears to persist even in larger samples (Camilo, Thorsett, \& Kulkarni 1994; Camilo 1996). It can be found from Figure 1 that the observed binary pulsars have two magnetic field strength distributions: the high field strength pulsars with $B_{s} \geq 10^{10} \mathrm{G}$, which consist of high-mass binary pulsars (HMBPs), and the low field strength pulsars with $B_{s} \leq 10^{9} \mathrm{G}$, which consist of the rapidly rotating low-mass binary pulsars (LMBPs), together with five seemly special low-mass binary pulsars. It can be conjectured that such a distribution could arise if two types of pulsars exist and have different magnetic field strength distributions. One kind of evolutionary models, referred to as the crustal ones, suggest accretion-induced field decay, in which different accretion histories for the LMXBs and the high-mass X-ray binaries (HMXBs) could give rise to different distributions (Taam \& van den Heuvel 1986; Shibazaki et al. 1989; Romani 1990). As these models argued, the higher total accreted mass should lead to the stronger field decay. Very recently van den Heuvel \& Bitzaraki (1995) analyzed the evolutionary process forming the LMBPs and further found that when the neutron stars accrete matter with mass more than $0.5 M_{\odot}$ their magnetic fields saturate at $\sim 3 \times 10^{8} \mathrm{G}$. Another kind of evolutionary models are the interior ones where interaction of the magnetic flux tubes with quantized vortices in the interior leads to field expulsion (Srinivasan et al. 1990). Ruderman (1991a, 1991b) developed a variant of an interior model, in which the rotation-magnetic-field coupling results in rearranging the magnetic flux lines, thus leading to changes in the dipole field

${ }^{1}$ Department of Physics, University of Hong Kong, Hong Kong.

2 Department of Astronomy, Nanjing University, Nanjing 210093, China. strength. Jahan Miri \& Bhattacharya (1994) proposed a model in which the spin-down of a neutron star leads to the expulsion of the magnetic flux outward to the crust. Once deposited in the crust, the magnetic field may decay because of ohmic dissipation. This model can give the bottom field $\sim 10^{8} \mathrm{G}$ if the ohmic timescale is assumed to be in the range $10^{8.5}-10^{9} \mathrm{yr}$. However, these values might be much less than computed for the deep layers of the inner crust (Sang \& Chanmugam 1987; Pethick \& Sahrling 1995). Thus, all of the models seem not to provide a natural explanation for the bimodal field distribution.

In this Letter we analyze the crustal physics of accreting neutron stars in HMXBs and LMXBs and argue that the crustal shear angle of neutron stars in LMXBs is much smaller than that of neutron stars in HMXBs. The bimodal field distribution may be understood by the fact that neutron stars in LMXBs and HMXBs have different physical properties of crusts that support different magnetic fields. In addition, we may provide an explanation for van den Heuvel \& Bitzaraki's (1995) result-why the magnetic field strengths of millisecond pulsars with low-mass companions saturate at $\sim 3 \times 10^{8} \mathrm{G}$ when accreted mass exceeds $\sim 0.5 M_{\odot}$.

\section{DESCRIPTION OF THE MODEL}

\subsection{Crustal Properties of Accreting Neutron Stars}

The inner crust of a neutron star consists of relativistic degenerate electrons, lattice nuclei, and nonrelativistic degenerate neutrons. It is usually expected that these lattice nuclei have a bcc structure with melting temperature

$$
T_{m}=\frac{(Z e)^{2}}{180 b_{z} k_{b}} \sim 4 \times 10^{9}\left(\frac{Z}{40}\right)^{5 / 3} \mathrm{~K}
$$

and shear modulus

$$
\mu=0.3 \frac{(Z e)^{2}}{b_{z}^{4}} \sim 2 \times 10^{29}\left(\frac{Z}{40}\right)^{2 / 3} \mathrm{dyn} \mathrm{cm}^{-2},
$$

where $b_{z}$ is the internuclear spacing, $k_{b}$ is Boltzmann's constant, and $Z$ is the atomic number of the nuclei. We have assumed the crust density $\rho \sim 5 \times 10^{13} \mathrm{~g} \mathrm{~cm}^{-3}$, near where the neutron vortex line pinning is strongest (Alpar et al. 1984).

In contrast, the shear angle $\theta$ of a lattice, defined as change in length per unit length under tension or compression, is dependent upon the ratio of the lattice temperature to the 


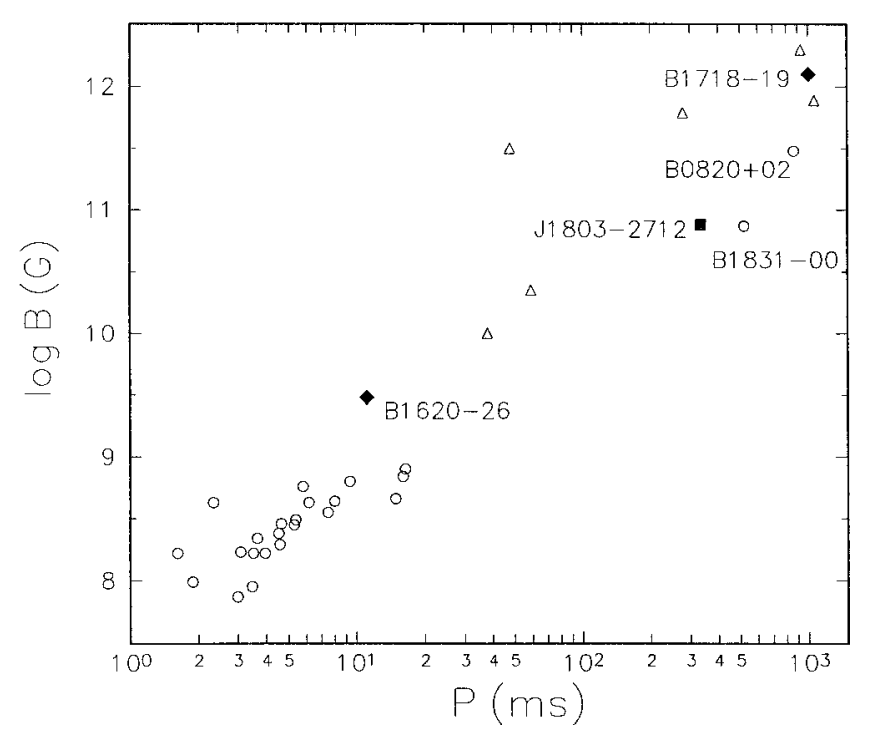

FIG. 1.-Surface dipole magnetic field strength vs. rotation period diagram of binary radio pulsars. Low-mass (circles) and high-mass (triangles) binary pulsars, respectively, which are not in any globular cluster. PSR J1803-2712 (square) is probably associated with SNR W30 (Taylor, Manchester, \& Lyne 1993). Diamonds: low-mass binary pulsars in globular clusters. PSRs B1831-00 and $\mathrm{B} 0820+02$ are discussed in the text.

melting temperature. This relation for neutron star crusts is usually extrapolated from stress experiments on ionic crystals at ordinary densities in the laboratory (Ruderman 1991a, 1991b). We adopt the following significant extrapolative features for neutron star crusts: $(a) \theta \sim 10^{-2}$ for $T / T_{m} \ll 0.1,(b)$ $\theta \sim 5 \times 10^{-3}$ at $T / T_{m} \sim 0.1$, and $(c) \theta \sim 10^{-3}$ for $0.1 \ll$ $T / T_{m}<1$ (Sidersleben \& Taylor 1989).

Neutron stars are generally believed to be born as very hot objects with a temperature greater than $10 \mathrm{MeV}$. At such a high temperature matter can be considered as being in a complete thermodynamic equilibrium with respect to the interactions between its constituents. Matter in which many exothermic reactions could be fully completed is usually referred to as catalyzed matter. For an isolated neutron star, its whole crust is constructed out of catalyzed matter, and therefore the shear angle of its inner crust should be $\theta \sim 10^{-2}$ when the star becomes rather cold. For an accreting neutron star, explosive burning of the helium layer leads to formation of matter consisting mainly of ${ }^{56} \mathrm{Ni}$, which transforms into ${ }^{56} \mathrm{Fe}$. The growing layer of processed accreted matter pushes down the original crust. Because the temperature in the crust is rather low (see eq. [5]), the only processes that can take place in crystallized matter when it moves inward are electron capture, neutron emission or absorption, and, at sufficiently high density, pycnonuclear fusion (Sato 1979; Haensel \& Zdunik 1990a). If the accretion rate is $\sim 10^{-10} M_{\odot} \mathrm{yr}^{-1}$ and the accretion timescale does not exceed $10^{7} \mathrm{yr}$, the original catalyzed matter in the deep layers of the inner crust is not replaced by the processed accreted matter. But if the accretion rate is $\sim 10^{-8} M_{\odot} \mathrm{yr}^{-1}$ and the accretion timescale exceeds $10^{8}$ $\mathrm{yr}$, the processed accreted matter must replace the original catalyzed matter of the inner crust. In these cases, even though accretion stops, the processed accreted matter cannot convert into catalyzed matter because the conversion is prohibited because of high Coulomb barriers (Haensel \& Zdunik 1990a). In order to determine the shear angle and modulus of the inner crust of accreting neutron star, one must know the crustal temperature.

The thermal structure of accreting neutron stars has been studied (see, e.g., Fujimoto et al. 1984; Miralda-Escudé, Haensel, \& Paczyński 1990). It was argued that, after a relatively short initial stage $\left(\leq 10^{5} \mathrm{yr}\right)$, the temperature distribution reaches a steady state. Then the temperature is practically uniform in deep layers of the crusts of neutron stars with standard neutrino emissivities. The value of the crustal temperature in a steady state is dependent on the accretion rate $\dot{M}$. Physically, this relation can be approximately obtained through the fact that the rate for energy released by nuclear reactions in the crust of an accreting neutron star is equal to that for energy radiated in neutrinos in the interior.

We first consider the heating processes in the crust of an accreting neutron star. As analyzed by Sato (1979) and Haensel \& Zdunik (1990a), the nuclear reactions by which energy is deposited in the crust of an accreting neutron star consist of electron capture, neutron emission (or absorption), and, at sufficiently high density, pycnonuclear fusion. These nonequilibrium processes release heat, and the total effective heat per unit time is approximated by

$$
Q=\eta \dot{M} c^{2},
$$

where $\eta$ is the efficiency factor for nuclear reactions $\left(\approx 2 \times 10^{-3}\right)$. Second, we take the cooling processes in the neutron star interior into account. Using standard cooling models for a neutron star, when its interior temperature is below $10^{10} \mathrm{~K}$, the emissivity for neutrino pair bremsstrahlung from nuclei in the crust dominates over that for modified Urca reactions in the core because the neutrons are "locked" in a superfluid state so that the rates for modified Urca reactions are cut down exponentially. According to Festa \& Ruderman (1969), the neutrino luminosity due to crustal neutrino pair bremsstrahlung is given by

$$
L_{v}^{\mathrm{cr}}=1.7 \times 10^{39} M_{*} f T_{9}^{6} \mathrm{ergs} \mathrm{s}^{-1},
$$

where $M_{*}$ is the neutron star mass in units of $1 M_{\odot}, f$ is the ratio of the crustal mass to the stellar total mass, and $T_{9}$ is the temperature in units of $10^{9} \mathrm{~K}$. Now let $Q$ be equal to $L_{\mathrm{cr}}$, and then we obtain the steady-state temperature in the interior

$$
T_{9} \approx 0.61\left(\frac{\eta_{-3} \dot{M}_{18}}{f_{-2} M_{*}}\right)^{1 / 6},
$$

where $\eta_{-3}=\eta / 10^{-3}$ and $f_{-2}=f / 10^{-2}$ and $\dot{M}_{18}$ is in units of $10^{18}$ $\mathrm{g} \mathrm{s}^{-1}$.

Therefore, if the neutron star in an X-ray binary accretes matter from its companion at $\sim 10^{16} \mathrm{~g} \mathrm{~s}^{-1}$ and the total accreted mass $\Delta M$ is much smaller than the crustal mass $M_{\mathrm{cr}}$, then there is only cold catalyzed matter in deep layers of the inner crust $(Z \sim 40)$ (Negele \& Vautherin 1973), and $T_{m} \sim 4 \times 10^{9} \mathrm{~K}$, in which case $T \approx 2.7 \times 10^{8} \mathrm{~K}<0.1 T_{m}$, and thus the crustal shear angle is about $10^{-2}$. But if the accretion rate of the neutron star is the Eddington rate $\left(\sim 10^{18} \mathrm{~g} \mathrm{~s}^{-1}\right)$ and $\Delta M>M_{\mathrm{cr}}$, then the original catalyzed matter is replaced by the processed accreted matter $(Z \sim 20)$ (Haensel \& Zdunik 1990b), and $T_{m} \sim 10^{9} \mathrm{~K}$. In this case $0.1 T_{m} \ll T \approx 6.1 \times 10^{8} \mathrm{~K}<T_{m}$, and thus the crustal shear angle is about $10^{-3}$. 


\subsection{Magnetic Field Evolution of Neutron Stars in $L M X B s$ and $H M X B s$}

We now turn to the study of magnetic field evolution of neutron stars in LMXBs and HMXBs. It is well known that when the temperature in the interior of a neutron star is smaller than $\sim 10^{10} \mathrm{~K}$, its core protons and neutrons, as expected, form a type II superconductor and superfluid (for a review, see Sauls 1989). The core magnetic field is organized into an array of quantized magnetic flux tubes that terminate near the base of the crust. There they open out and traverse the crust as a normal magnetic field. It has been pointed out that because of the superfluid-drag effect, a nonvanishing superfluid neutron velocity will generate a charge current around each vortex (Alpar, Langer, \& Sauls 1985). The induced supercurrent will result in a strong magnetic field associated with each neutron vortex. Both vortex and flux tube contain an average magnetic field $B_{c} \sim 10^{15} \mathrm{G}$ (Sauls 1989). As the neutron star spins up, vortex lines will carry the flux tubes and cause them to move inward (Srinivasan et al. 1990; Ruderman 1991a, 1991b; Ding, Cheng, \& Chau 1993). We have to point out that the detailed motion of the flux tubes inside the stellar core is a very complicated issue. We will not discuss this problem any further. According to the plate tectonics model (Ruderman 1991a, 1991b), the magnetic field lines of the star may be finally pushed into a small cap around the spin axis. The magnetic field becomes so strong that it breaks the crust until the strength of the magnetic field is

$$
\begin{aligned}
B \sim & 5 \times 10^{12} \mathrm{G}\left(\frac{\theta}{10^{-2}}\right)\left(\frac{\mu}{2 \times 10^{29} \mathrm{dyn} \mathrm{cm}^{-2}}\right) \\
& \times\left(\frac{l}{10^{5} \mathrm{~cm}}\right)\left(\frac{R}{10 \mathrm{~km}}\right)^{-1},
\end{aligned}
$$

where $l$ is the crustal thickness, $R$ is the stellar radius, and $\mu$ is the shear modulus at the density $\sim 5 \times 10^{13} \mathrm{~g} \mathrm{~cm}^{-3}$. This expression is obtained by setting the maximum shear stress $(\sim \mu \theta l / R)$, which the crust could bear before yielding, equal to the shear stress on the crustal base $\left(\sim B B_{c} / 8 \pi\right)$. Since the observable dipole moment $\left(\sim B_{\mathrm{ob}} R^{3}\right)$ should be equal to the magnetic moment in the small cap $\left(\sim B l^{2} R\right)$, we obtain the dipole magnetic field strength:

$$
\begin{aligned}
B_{\mathrm{ob}} \sim & 5 \times 10^{10} \mathrm{G}\left(\frac{\theta}{10^{-2}}\right)\left(\frac{\mu}{2 \times 10^{29}{\mathrm{dyn} \mathrm{cm}^{-2}}^{-3}}\right. \\
& \times\left(\frac{l}{10^{5} \mathrm{~cm}}\right)^{3}\left(\frac{R}{10 \mathrm{~km}}\right)^{-3} .
\end{aligned}
$$

We assume that the mass of a neutron star before accretion is $1.4 M_{\odot}$. In HMXB, the neutron star possibly accretes matter from its companion at $\sim 10^{-10} M_{\odot} \mathrm{s}^{-1}$ by a strong wind (Ögelman 1989; Bhattacharya \& van den Heuvel 1991), and $\Delta M \ll M_{\text {cr }}$ because of the short life time of the companion. Thus, the deep layers of the inner crust of the neutron star remain an ideal Coulomb lattice with $\theta \sim 10^{-2}$ and $\mu \sim 2 \times 10^{29}$ dyn $\mathrm{cm}^{-2}$. On the other hand, an LMXB is presumably kept in a steady mass-transfer state throughout the evolutionary timescale of the companion at a rate $\sim 10^{-8} M_{\odot}$ $\mathrm{s}^{-1}$. Thus, such an X-ray binary can be a luminous X-ray source for $10^{8}$ years or more (Ögelman 1989; Bhattacharya \& van den Heuvel 1991). If so, the deep layers of the inner crust of the neutron star should have $\theta \sim 10^{-3}, \mu \sim 1.3 \times 10^{29}$ dyn $\mathrm{cm}^{-2}$
TABLE 1

Stellar Structure and Observable Magnetic Fields

\begin{tabular}{ccccc}
\hline \hline EOS & $\begin{array}{c}R \\
(\mathrm{~km})\end{array}$ & $\begin{array}{c}M_{\mathrm{cr}} \\
\left(M_{\odot}\right)\end{array}$ & $\begin{array}{c}l \\
\left(10^{5} \mathrm{~cm}\right)\end{array}$ & $\begin{array}{c}B_{\mathrm{ob}} \\
(\mathrm{G})\end{array}$ \\
\hline \multicolumn{5}{c}{ Neutron Stars with $1.4 M_{\odot}$} \\
WFF $1 \ldots \ldots \ldots \ldots$ & 10.4 & 0.028 & 0.82 & $2.5 \times 10^{10}$ \\
WFF $2 \ldots \ldots \ldots \ldots$ & 11.1 & 0.040 & 1.06 & $4.4 \times 10^{10}$ \\
FP $\ldots \ldots \ldots \ldots \ldots$ & 10.6 & 0.031 & 0.88 & $2.8 \times 10^{10}$ \\
BJ $\ldots \ldots \ldots \ldots \ldots$ & 11.5 & 0.074 & 1.78 & $1.9 \times 10^{11}$ \\
PS $\ldots \ldots \ldots \ldots \ldots$ & 15.0 & 0.404 & 4.40 & $1.3 \times 10^{12}$ \\
\hline
\end{tabular}

Neutron Stars with $1.9 M_{\odot}$

\begin{tabular}{lllll}
\hline WFF $1 \ldots \ldots \ldots \ldots$ & 10.2 & 0.014 & 0.43 & $3.7 \times 10^{8}$ \\
WFF $2 \ldots \ldots \ldots$ & 10.8 & 0.020 & 0.55 & $6.5 \times 10^{8}$ \\
FP $\ldots \ldots \ldots \ldots \ldots$ & 10.0 & 0.013 & 0.41 & $3.5 \times 10^{8}$ \\
BJ $\ldots \ldots \ldots \ldots \ldots$ & 10.2 & 0.024 & 0.73 & $1.8 \times 10^{9}$ \\
PS $\ldots \ldots \ldots \ldots \ldots$ & 14.8 & 0.256 & 3.62 & $7.4 \times 10^{10}$
\end{tabular}

NoTE.-Columns (1)-(5) represent selected equations of state, stellar radii, crustal masses, crustal thicknesses, and magnetic field strengths calculated from eq. (7), respectively.

when the mass of a low-mass binary pulsar now is $\sim 1.9 \mathrm{M}_{\odot}$. Therefore, from equation (7), the observed magnetic field strengths of high-mass and low-mass binary pulsars are determined by the shear angle as well as the crustal thicknesses and radii of neutron stars with $1.4 M_{\odot}$ and $1.9 M_{\odot}$, respectively.

In general, for a given mass, stellar models with stiffer equations of state (EOSs) have thicker crusts with larger mass. We select the following EOSs: BJ (Reid potential with shortrange repulsion and hyperons) (Bethe \& Johnson 1974), FP (three-nucleon interaction) (Friedman \& Pandharipande 1981), WFF1 (AV14+UVII) (Wiringa, Fiks, \& Fabrocini 1988), WFF2 (UV14+UVII) (Wiringa et al. 1988), and PS (mean field) (Pandharipande \& Smith 1975). The first four EOSs are moderately stiff, while the final EOS is stiff. We do not consider soft EOSs for the following reasons. First, some soft EOSs at high densities are ruled out by the postglitch recovery in four pulsars (Link, Epstein, \& Van Riper 1992). Second, an EOS such as kaon condensation may not occur in stable neutron stars (Pandharipande, Pethick, \& Thorsson 1995). Third, if the EOSs in the core of a neutron star in LMXB before accretion were soft, the $1.9 M_{\odot}$ compact object after accretion would be a black hole (Brown 1988). From equation (7), we show the observed magnetic field strengths of neutron stars with the above five EOSs in Table 1. It can be seen that if EOSs at high densities are moderately stiff, two types of pulsars formed in HMXBs and LMXBs have different effective magnetic field strengths, which may explain the observed bimodal field distribution (Kulkarni 1992; Camilo et al. 1994; Phinney \& Kulkarni 1994).

In the above, we have assumed that when the mass of the neutron star in LMXB is about $1.9 M_{\odot}$ its interior is normal neutron matter. As argued by Cheng \& Dai (1996), however, such a massive neutron star may convert to a strange star, which is suggested as a possible origin of cosmological $\gamma$-ray bursts. In the absence of a superconducting core in a strange star, the magnetic stress at its crustal base, which is at the neutron-drip density, might plausibly become $B^{2} / 8 \pi$. Thus, we can calculate the observable magnetic field strength $B_{\text {ob }} \sim 4 \times 10^{8} \mathrm{G}(l / 150 \mathrm{~m})^{5 / 2}(R / 10 \mathrm{~km})^{-5 / 2}$. We want to point out that the bottom field predicted by this scenario will be 
independent of the accretion rate, which differs from the previous scenario.

In both scenarios, the crustal parameters change little after the amount of accreted mass exceeds $0.5 M_{\odot}$. Thus, the stellar magnetic field is unlikely to vary significantly. This seems to provide an explanation of why the observed magnetic fields of millisecond pulsars with low-mass companions may saturate at $\sim 3 \times 10^{8} \mathrm{G}$ when their accreted masses exceed $\sim 0.5 M_{\odot}$.

\section{DISCUSSION AND CONCLUSION}

We discuss five seemingly special pulsars: PSRs B1718-19, B1620-26, B1831-00, B0820+02, and J1803-2712 (Camilo 1996; ven den Heuvel \& Bitzaraki 1995). The first two pulsars are in the globular clusters, and they may be the result of recent capture events and have possibly accreted only very little. For PSRs B1831-00 and B0820+02, if their present periods are assumed to be the rebirth ones, then their accretion rates in the accretion phase are $\sim 2 \times 10^{17} \mathrm{~g} \mathrm{~s}^{-1}$ and $2 \times 10^{18} \mathrm{~g} \mathrm{~s}^{-1}$, respectively, and thus their crustal shear angles are about $10^{-3}$. For PSR B1831-00, since the amount of its accreted mass is $\sim 0.8 M_{\odot}$ (van den Heuvel \& Bitzaraki 1995), its crustal thickness is about 2.5 times less than that of the 1.4 $M_{\odot}$ neutron star with the same EOS. If the initial field configuration is further assumed to be that its north and south magnetic polar caps are in opposite hemispheres, then the result of the crustal plate motion is that these two polar caps will never end up at the same hemisphere (Ruderman 1991a, 1991b). Therefore, from equation (6), we find that the effective magnetic field strength of PSR $1831-00$ is $B_{\mathrm{ob}} \sim 2 \times 10^{11}$ $\mathrm{G}(l / 250 \mathrm{~m})(R / 10 \mathrm{~km})^{-1}$, where $\theta$ and $\mu$ have been taken to be $10^{-3}$ and $1.3 \times 10^{29} \mathrm{dyn}^{\mathrm{cm}}{ }^{-2}$, respectively. For stellar models with moderately stiff EOSs, this strength is close to the observed value $\left(\sim 8.7 \times 10^{10} \mathrm{G}\right)$. For PSR B0820+02, the amount of its accreted mass is only $0.04 M_{\odot}$ (van den Heuvel \& Bitzaraki 1995), according to equation (6), its magnetic field is about $5 \times 10^{11}\left(l / 10^{5} \mathrm{~cm}\right)(R / 10 \mathrm{~km})^{-1} \mathrm{G}$, which is consistent with the observed value $\left(\sim 3 \times 10^{11} \mathrm{G}\right)$. PSR J1803-2712 is probably associated with supernova remnant (SNR) W30 (Taylor, Manchester, \& Lyne 1993), and its magnetic field strength cannot be estimated from our model because this pulsar may be formed by accretion-induced collapse.

PSR B1831-00 has been discussed by some authors. Phinney \& Kulkarni (1994) suggested a model in which following the supernova explosion the neutron star of 1831-00 was shot into its normal companion, due to a large kick velocity, so that this companion was almost destroyed, and only a small amount, $<0.2 M_{\odot}$, was left. In order to make this model work, van den Heuvel \& Bitzaraki (1995) argued that the neutron star should be formed by accretion-induced collapse. Thus, this pulsar appears to have a strong magnetic field strength.

Our model can predict that the observed sample of binary pulsars should contain a majority of low-field pulsars. This is because if the initial two field poles of a neutron star are in opposite hemispheres, then according to the crustal plate tectonics the dipole moment becomes aligned by spin-up (Ruderman 1991a, 1991b). Such a neutron star cannot be observed as a pulsar because of the absence of radio pulses. Therefore, this observational selection effect will reduce the number of high-field binary pulsars.

In summary, we have analyzed the crustal physics of accreting neutron stars in HMXBs and LMXBs and argued that the crustal shear angle of neutron stars in LMXBs is much smaller than that of neutron stars in HMXBs. We have found that the bimodal field distribution of pulsars may be understood by the fact that neutron stars in LMXBs and HMXBs have different physical properties of crusts that support different magnetic fields. In addition, we may provide an explanation for van den Heuvel \& Bitzaraki's (1995) result-why the magnetic field strengths of millisecond pulsars with low-mass companions saturate at $\sim 3 \times 10^{8} \mathrm{G}$ when accreted mass exceeds $\sim 0.5 M_{\odot}$.

We thank M. A. Ruderman for bringing this problem to our attention and F. K. Lamb for enlightening discussions. Part of this work begins at Aspen Center for physics and is supported by the University and Polytechnics Grant Committee grant of Hong Kong. Z. G. D. thanks the National Natural Committee of China for support.

\section{REFERENCES}

Alpar, M. A., Anderson, P. W., Pines, D., \& Shaham, J. 1984, ApJ, 276, 325

Alpar, M. A., Langer, S., \& Sauls, J. 1985, ApJ, 282, 533

Bethe, H. A., \& Johnson, M. 1974, Nucl. Phys. A, 230, 1

Bhattacharya, D., \& van den Heuvel, E. P. J. 1991, Phys. Rep., 203,

Brown, G. E. 1988, Nature, 336, 519

Camilo, F. 1996, preprint

Camilo, F., Thorsett, S. E., \& Kulkarni, S. R. 1994, ApJ, 421, L15

Cheng, K. S., \& Dai, Z. G. 1996, Phys. Rev. Lett., 77, 1210

Ding, K. Y., Cheng, K. S., \& Chau, H. F. 1993, ApJ, 408, 167

Festa, G. G., \& Ruderman, M. A. 1969, Phys. Rev., 180, 1227

Friedman, B., \& Pandharipande, V. R. 1981, Nucl. Phys. A, 361, 502

Fujimoto, M. Y., Hanawa, T., Iben, I., \& Richardson, M. B. 1984, ApJ, 278, 813

Haensel, P., \& Zdunik, J. L. 1990a, A\&A, 227, 431

1990b, A\&A, 229, 117

Jahan Miri, M., \& Bhattacharya, D. 1994, MNRAS, 269, 455

Kulkarni, S. R. 1992, Philos. Trans. R. Soc. London, A, 341, 77

Link, B., Epstein, R. I., \& Van Riper, K. A. 1992, Nature, 359, 616

Miralda-Escudé, J., Haensel, P. \& Paczyński, B. 1990, ApJ, 362, 572

Negele, J., \& Vautherin, D. 1973, Nucl. Phys. A, 207, 298

Ögelman, H. 1989, in Timing Neutron Stars, ed. H. Ögelman \& E. P. J. van den Heuvel (Dordrecht: Kluwer), 169
Pandharipande, V. R., Pethick, C. J., \& Thorsson, V. 1995, Phys. Rev. Lett., 75, 4567

Pandharipande, V. R \& Smith, R. A. 1975, Phys. Lett., B59, 15

Pethick, C. J., \& Sahrling, M. 1995, ApJ, 453, L29

Phinney, E. S., \& Kulkarni, S. R. 1994, ARA\&A, 32, 591

Romani, R. W. 1990, Nature, 347, 741

Ruderman, M. A. 1991a, ApJ, 366, 261

- 1991b, ApJ, 382, 576

Sang, Y., \& Chanmugam, G. 1987, ApJ, 323, L61

Sato, K. 1979, Prog. Theor. Phys., 62, 957

Sauls, J. 1989, in Timing Neutron Stars, ed. H. Ögelman \& E. P. J. van den Heuvel (Dordrecht: Kluwer), 457

Shibazaki, N., Murakami, T., Shaham, J., \& Nomoto, K. 1989, Nature, 342, 656 Sidersleben, M., \& Taylor, G. 1989, Philos. Mag. A., 60, 631

Srinivasan, G., Bhattacharya, D., Muslimov, A. G., \& Tsygan, A. I. 1990, Curr. Sci., 59,31

Taam, R. E., \& van den Heuvel, E. P. J. 1986, ApJ, 305, 235

Taylor, J. H., Manchester, R. N., \& Lyne, A. G. 1993, ApJS, 88, 529

van den Heuvel, E. P. J., \& Bitzaraki, O. 1995, A\&A, 297, L41

Wiringa, R. B., Fiks, V., \& Fabrocini, A. 1988, Phys. Rev., C38, 1010 